Pathologe 2009 · 30:74

DOI 10.1007/s00292-008-1116-x

Online publiziert:18. Dezember 2008

๑) Springer Medizin Verlag 2008

N. Freudenberg

Institut für Pathologie, Freiburg

\title{
Offener Brief der Deutschen Gesellschaft für Zytologie
}

\section{An die Deutsche Gesellschaft für Gynäkologie und Geburtshilfe}

Sehr geehrter Herr Prof. Jonat, sehr geehrter Herr Prof. Friese, sehr geehrter Herr Prof. Beckmann,

uns liegt die Leitlinie 2.4.4. „Prävention, Diagnostik und Therapie der HPVInfektion und präinvasiver Läsionen des weiblichen Genitale AWMF 015/O27“ der Deutschen Gesellschaft für Gynäkologie und Geburtshilfe (DGGG) vor. Mit Bedauern haben wir festgestellt, dass die Deutsche Gesellschaft für Zytologie (DGZ) bei der Erstellung dieser Leitlinie übergangen wurde.

Die derzeitige gesetzliche Krebsvorsorge bzw. Früherkennung mit Zervixabstrichen wird zum größten Teil von Mitgliedern unserer Gesellschaft erbracht. Zytologen sind bei der Beratung der in der Vorsorge aktiven Gynäkologen entscheidend tätig. Die DGGG hat somit eine Leitlinie auf der Basis der Meinung einer nichtrepräsentativen Expertenselektion erstellt.

Die Validität dieser Leitlinie und ihre Einordnung als S2k-Leitlinie ist damit infrage gestellt. Zudem wurden vorhandene Interessenkonflikte nicht dargelegt, was den Wert dieser Leitlinie weiter reduziert. $\mathrm{Zu}$ zahlreichen Aspekten wurden Empfehlungen ohne Angabe einer Evidenz ausgesprochen.
Die DGGG hat sich unseres Erachtens mit dieser Leitlinie von dem sehr erfolgreichen Vorsorgeprogramm in Deutschland distanziert und scheint auf nichtvalidierte Konzepte zu setzen.

Wir bitten Sie, auch im Einvernehmen mit der Arbeitsgemeinschaft der Wissenschaftlichen Medizinischen Fachgesellschaften (AWMF) zu klären, wie mit der Leitlinie weiter zu verfahren ist. In der vorliegenden Form lehnt die DGZ diese Leitlinie ab.

Mit freundlichen Grüßen

Prof. Dr. Dr. h.c. Nikolaus Freudenberg

\section{Korrespondenzadresse}

Prof. Dr. Dr. h.c. N. Freudenberg

Institut für Pathologie

Breisacher Str. 115a, 79106 Freiburg

nikolaus.freudenberg@uniklinik-freiburg.de 